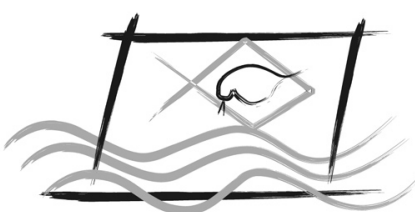

ECOTOX - BRASIL

\title{
Estudo sazonal de metais pesados no sedimento do Saco do Laranjal - Pelotas-RS
}

\author{
G. R. Betemps ${ }^{1} \&$ P. J. SAnches Filho ${ }^{1}$ \\ ${ }^{1}$ Grupo de Pesquisa em Contaminantes Ambientais - GPCA, Instituto Federal Sul-rio-grandense, Praça Vinte de Setembro, 455, CEP \\ 96015-360, Pelotas-RS, Brasil.
}

(Received Septemper 21, 2010; Accept Septemper 27, 2011)

\begin{abstract}
Resumo
O Saco do Laranjal é referido pela Base de Dados Tropicais (BDT) como uma área prioritária para a preservação. Pouco se conhece sobre a geoquímica dos sedimentos da região de estudo, tornando-se indispensável para as ações de monitoramento a determinação de metais pesados no sedimento. Nesse contexto, foi desenvolvido o estudo dos metais pesados como $\mathrm{Cu}, \mathrm{Pb}, \mathrm{Zn}$ e Cr no sedimento do Saco do Laranjal, porção estuarina da Laguna dos Patos. A amostragem percorreu seis pontos do local de estudo, nos períodos de inverno e primavera de 2009 e verão de 2010. As amostras foram extraídas em triplicata com água régia a $50 \%$ e ácido perclórico e analisadas através de espectrometria de absorção atômica em chama. Neste estudo também foram avaliados a matéria orgânica por método indireto através da perda de voláteis e, granulometria. Os resultados demonstram baixos índices de contaminação comparados com o Guia de Qualidade para Sedimentos Estuarinos Canadense, apresentando estreita relação entre o aumento da matéria orgânica no teor de finos (fração $<63 \mu \mathrm{m}$ ) e a concentração dos metais.
\end{abstract}

Palavras chave: estuário, sazonalidade, matéria orgânica, análise de metais.

Seasonal study of heavy metals in sediment from Saco do Laranjal - Pelotas -RS

\begin{abstract}
The Saco do Laranjal is referred by the Tropical Database (BDT) as a priority area for preservation. However, little is known about the geochemistry of the sediments of the study area, making the determination of heavy metals in the sediment of Saco do Laranjal indispensable for monitoring actions. The study of heavy metals such as $\mathrm{Cu}, \mathrm{Pb}, \mathrm{Zn}$ and $\mathrm{Cr}$ in sediments of Saco do Laranjal, a estuarine portion of the Patos Lagoon was performed. The sampling was done in six points of the study site during periods of winter and spring 2009 and summer 2010. The samples were extracted in triplicate with 50\% aqua-regia and perchloric acid, and analyzed by flame atomic absorption spectrometry. This study also evaluated the organic matter by the indirect method of loss of volatiles, and granulometry. The results demonstrated low contaminations rates compared with the Canadian Quality Guide for Estuarine Sediments, showing a close relationship between the increase in organic matter of the fines content (fraction $<63 \mu \mathrm{m}$ ) and the concentration of the metals.
\end{abstract}

Keywords: estuary, seasonality, organic matter, metal analysis. 


\section{INTRODUÇÃO}

Pelotas, cidade pólo no Sul do Brasil, localizada junto à Laguna dos Patos, conta com cerca de 320.000 habitantes. A maioria da população, $93,17 \%$ do total, está localizada na zona urbana. O Saco do Laranjal, situado na Laguna dos Patos, apresenta-se dividido em diversas praias: Pontal da Barra, Laranjal, Barro Duro, Totó e Colônia Z3. Junto à saída do Canal São Gonçalo encontra-se a praia do Pontal da Barra, essa região é referida pela Base de Dados Tropicais (BDT) como uma área prioritária para preservação, uma vez que se constitui em berçário para criação de tainhas, sendo fonte de subsistência de centenas de famílias (RAMB, 2003).

Os estuários apresentam grande produtividade, e os padrões biológicos e produtivos do ecossistema são influenciados diretamente pela ação das chuvas, salinidade e vento, e também pelo carreamento de nutrientes inorgânicos provindos do continente, entrada das águas do mar, efluentes domésticos e de atividades agrícolas (Mirlean et al. 2003). As lagunas têm características expressivamente diferentes de outros tipos de estuários porque representam uma porção significativa da interface mar-terra (Windom et al, 1999). Pouco se conhece sobre a geoquímica dos sedimentos da região de estudo, tornando-se indispensável para as ações de monitoramento a determinação dos teores de contaminantes traços como metais pesados no sedimento.

A definição do termo "metais pesados" tem sido muito discutida na literatura, no entanto, Castro (2006) cita diversos autores que através de suas definições consideram metais pesados os elementos propostos neste estudo.

Nos ecossistemas aquáticos, os metais que têm função biológica participam nos processos fisiológicos dos organismos aquáticos, os que não têm são geralmente tóxicos a uma grande variedade de espécies. Mesmo aqueles metais com função biológica definida, quando em concentrações acima das normalmente encontradas no ambiente, podem apresentar toxicidade aos organismos vegetais e animais (Esteves, 1988). Os metais pesados são elementos não degradáveis e estão associados a efeitos nocivos no meio ambiente marinho e estuarino, pois apresentam toxicidade, persistência e bioacumulação na cadeia trófica (Lacerda \& Marins, 2006). A inclusão desses metais pode ocorrer através de processos de adsorção e complexação, apresentando ampla capacidade de acumulação em sedimentos de granulometria fina e com índices elevados de matéria orgânica (Lacerda \& Marins, 2006 apud Torres et al., 2008). A necessidade de se conhecer a fração quimicamente ativa do sedimento tem levado vários pesquisadores a utilizarem frações finas (< $63 \mu \mathrm{m}$ ) para amostragem e análises (Lemes et al. 2003).

Neste trabalho buscou-se investigar a distribuição dos metais $\mathrm{Cu}, \mathrm{Cr}, \mathrm{Pb}$ e $\mathrm{Zn}$ em sedimentos de forma sazonal, ao longo do Saco do Laranjal, com o principal objetivo de realizar um diagnóstico da qualidade do sedimento do Saco do Laranjal. Pretende-se desta forma estabelecer os níveis atuais destes contaminantes e ainda, traçar um perfil de comportamento dos mesmos, estabelecendo possíveis relações com os demais parâmetros analisados e as referências de qualidade do sedimento estuarino citadas.

\section{MATERIAL E MÉTODOS}

Fez-se amostragem simples nos períodos de inverno (Julho) e primavera (Novembro) de 2009 e verão (Março) de 2010, em seis pontos distribuídos ao longo do Saco do Laranjal: Ponto 1 (P1) - Barra do Canal São Gonçalo; Ponto 2 (P2) Praia do Laranjal - Balneário Valverde; Ponto 3 (P3) - Praia do Laranjal - Balneário Sto Antônio; Ponto 4 (P4) - Praia do Barro Duro; Ponto 5 (P5) - Praia do Totó - Eco-camping; Ponto 6 (P6) - Colônia Z-3. Cada ponto corresponde ao ponto médio do local de amostragem e a escolha dos locais de coleta se deve ao fato de serem bastante representativos com relação ao aporte de contribuições antrópicas ao Saco do Laranjal. Todos os pontos foram devidamente registrados com o auxílio de um GPS (Global Positioning System) modelo GARMIN Etrex Vista ${ }^{\circledR} \mathrm{H}$ (Fig. 1).

O sedimento superficial ( $0-5 \mathrm{~cm}$ profundidade) foi coletado, utilizando-se uma draga de aço inoxidável do tipo "Van Veen". O material da parte central da draga foi retirado e armazenado em pote de polietileno, previamente descontaminado. Após a coleta, as amostras foram transportadas para o laboratório e armazenadas sob temperatura de refrigeração a $\pm 4^{\circ} \mathrm{C}$. Os materiais utilizados no tratamento e armazenamento das amostras foram descontaminados, em uma solução de $\mathrm{HNO}_{3}$ a $10 \%(\mathrm{v} / \mathrm{v})$, por $24 \mathrm{hs}$ e em seguida secos em estufa a $105^{\circ} \mathrm{C}$ (Teódulo et al. 2003).

A extração dos metais foi realizada através de extração ácida. As amostras de sedimentos foram secas em estufa a $60^{\circ} \mathrm{C}$ por 48 horas, a fim de evitar o arraste dos metais pelo vapor de água. Em seguida, foram maceradas em almofariz e peneiradas. A fração $<63 \mu \mathrm{m}$ foi utilizada para o tratamento químico de extração. Pesou-se cerca de $2 \mathrm{~g}$ de cada sedimento em triplicata, adicionando-se $8 \mathrm{~mL}$ de água régia $50 \%\left(3: 1 \mathrm{HCl}: \mathrm{HNO}_{3}\right)$ e 1 $\mathrm{mL}$ de $\mathrm{HClO}_{4}$, aquecendo-se por $30 \mathrm{~min}$ a $90^{\circ} \mathrm{C}$ em BanhoMaria (Hortellani et al. 2008, procedimento com adaptações). A solução resultante foi filtrada e avolumada a $50 \mathrm{~mL}$ com água Milli-Q. Os padrões foram preparados por diluição a partir de soluções padrões marca Titrisol ${ }^{\mathbb{R}}$ Merck (1000 mg $\mathrm{L}^{-1}$ ). Durante o procedimento de extração foram utilizados frascos fechados para evitar perdas por volatilização. Todos os padrões foram submetidos ao mesmo tratamento químico das amostras para as perdas serem as mesmas, mantendo a proporcionalidade entre o sinal analítico e a concentração. Os extratos foram submetidos a análises por espectrometria de absorção atômica em chama em um espectrômetro GBC 932 Plus, as condições operacionais no equipamento de absorção atômica estão especificadas na tabela 1 (Tabela 1).

Em paralelo a cada extração foram realizadas análises de brancos. Para avaliação da exatidão foi utilizada a amostra de referência de metais para solo e sedimento Natural Matrix Certified Reference - Materials metals on soil/sediment \#4 (NMCR\#4), 

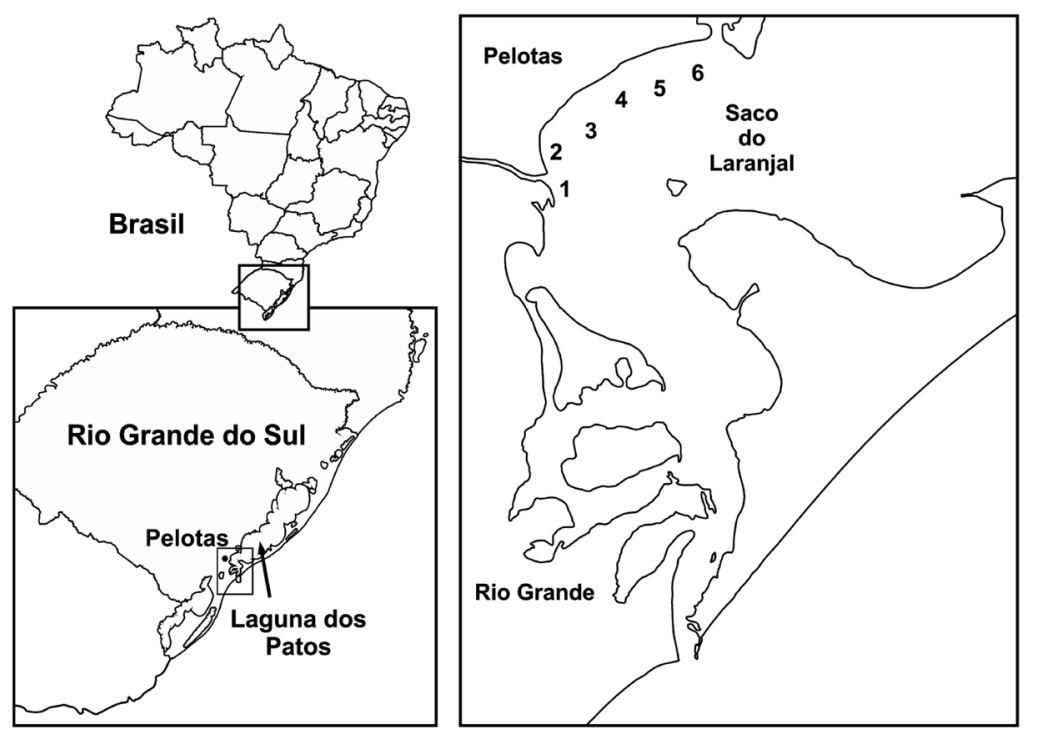

\begin{tabular}{|c|c|c|}
\hline \multicolumn{3}{|c|}{ GEOREFERÊNCIAS } \\
\hline P1 & W 52012'48.47" & S 31ㅇㄱ'08.81" \\
\hline P2 & W 52 $12^{\circ} 5$ & S 31046'10.31" \\
\hline P3 & W 52012'36.50" & S 314'15.79" \\
\hline $\mathrm{P} 4$ & W 52011'49.23" & S 314'26.71' \\
\hline P5 & W 52¹0'50.27" & S $31^{\circ} 43^{\prime} 47.84^{\prime \prime}$ \\
\hline$P 6$ & '08'22.62" & $S 31^{\circ} 42^{\prime} 38$. \\
\hline
\end{tabular}

Figura 1. Pontos de coleta e georeferências.

Tabela 1 - Condições operacionais utilizadas no espectrômetro de absorção atômica para a análise dos metais.

\begin{tabular}{lcccc}
\hline Elemento & Comprimento de onda & Largura da fenda & Energia da lâmpada & Gás-combustível \\
$\mathrm{Cu}$ & $324,7 \mathrm{~nm}$. & $0,5 \mathrm{~nm}$. & $3,0 \mathrm{~mA}$. & Ar-acetileno \\
$\mathrm{Cr}$ & $425,4 \mathrm{~nm}$. & $0,2 \mathrm{~nm}$. & $6,0 \mathrm{~mA}$. & $\mathrm{N}_{2} \mathrm{O}$-acetileno \\
$\mathrm{Pb}$ & $217,0 \mathrm{~nm}$. & $1,0 \mathrm{~nm}$. & $5,0 \mathrm{~mA}$. & Ar-acetileno \\
$\mathrm{Zn}$ & $213,9 \mathrm{~nm}$. & $0,5 \mathrm{~nm}$. & $5,0 \mathrm{~mA}$. & Ar-acetileno \\
\hline
\end{tabular}

adquirido da Ultra Scientific Analytical Solutions. Através de dez leituras do branco o limite de detecção (LOD) foi calculado usando a soma da média do sinal do branco mais três vezes o seu desvio padrão, enquanto que o limite de quantificação (LOQ) foi calculado pela soma da média do sinal do branco mais dez vezes seu desvio padrão (IUPAC 1997). Os ácidos $\mathrm{HCl}$ e $\mathrm{HNO}_{3}$ utilizados nas análises foram todos de grau analítico adquiridos da Merck, com exceção do $\mathrm{HClO}_{4}$, de marca Vetec.

Com o propósito de descrever as características físicas do sedimento e de possíveis fatores de influência sobre a distribuição dos metais, a quantificação do conteúdo de matéria orgânica foi avaliada indiretamente por perda de voláteis a $550^{\circ} \mathrm{C}$ por $4 \mathrm{~h}$, segundo metodologia padrão (APHA, 2005). A análise granulométrica do sedimento foi determinada por peneiramento para os sedimentos grosseiros, classificando os tamanhos de grãos de acordo com a escala de Wentworth. O método utilizado nesta análise foi descrito por Suguio (1973).

\section{RESULTADOS E DISCUSSÃO}

No período de estudo o Saco do Laranjal apresentouse atípico devido às mudanças climáticas na região. A condutividade elétrica da água, medida "in situ" com condutivímetro Instrutherm modelo CD-830, apresentou valores entre 540 e $11280 \mu \mathrm{S} \mathrm{cm}^{-1}$ no inverno (julho 2009), resultados considerados altos devido à entrada de água salgada no estuário. Na primavera (novembro 2009), em função de altos índices de precipitações pluviométricas, atingindo $351,5 \mathrm{~mm}$ no mês, obteve-se condutividades entre 80,77 e $713,5 \mu \mathrm{S} \mathrm{cm}^{-1}$ e, no verão (março 2010), foram anotados valores entre 94,82 e $980,0 \mu \mathrm{S} \mathrm{cm}^{-1}$, considerada baixa em relação ao inverno. Essas variações de condutividade podem ser associadas a quantidades de chuvas ocorridas na região durante a primavera e verão. Os metais detectados em todos os pontos foram associados à matéria orgânica e ao teor de finos (fração $<63 \mu \mathrm{m}$ ). Em relação às características hidrodinâmicas, por se tratar de um saco, a possibilidade de concentração dos contaminantes aumenta.

No período de inverno e primavera o ponto 1 , que representa a foz do Canal São Gonçalo junto à Laguna dos Patos, apresentou maiores níveis de metais, bem como os níveis de matéria orgânica eo teor de finos, com exceção do chumbo detectado em concentrações significativas na maioria dos pontos. No verão houve migração da área de maior concentração, passando a se detectar maiores níveis no ponto 3, situado no Balneário Santo Antônio, o que pode estar associado ao aumento de matéria orgânica devido ao deslocamento da população para o litoral no período de veraneio. Os níveis dos metais estudados, exceto o chumbo em alguns pontos, encontramse abaixo do background do estuário da Laguna dos Patos definido por Niencheski et al. (2002). Este fato deve ser atribuído à ausência de atividades industriais na região. Os resultados demonstram baixos índices de contaminação comparados com o Guia de Qualidade dos Sedimentos Estuarinos do Canadá (CCME EPC98E, 1999) (Tabela 2). 
Tabela 2 - Teores de matéria orgânica (MO\%) e respectivos desvios-padrão, níveis de metais em $\mathrm{mg} \mathrm{Kg}^{-1}$ e respectivos desvios-padrão, ISQG- Índice de qualidade do sedimento e PEL- Valor acima do qual efeito adverso é esperado em mg Kg${ }^{-1}$ (CCME EPC- 98E, 1999), BG - Background L. Patos em mg Kg-1 (Niencheski et al., 2002), coeficiente angular, b- coeficiente linear, LOD- limite de detecção e LOQ - limite de quantificação em $\mathrm{mg} \mathrm{Kg}^{-1}$. Valor certificado e intervalo de confiança do valor certificado em $\mathrm{mg} \mathrm{Kg}^{-1}$, valor obtido em $\mathrm{mg} \mathrm{Kg}^{-1}$ e respectivos desvios-padrão e recuperações (Rec. \%).

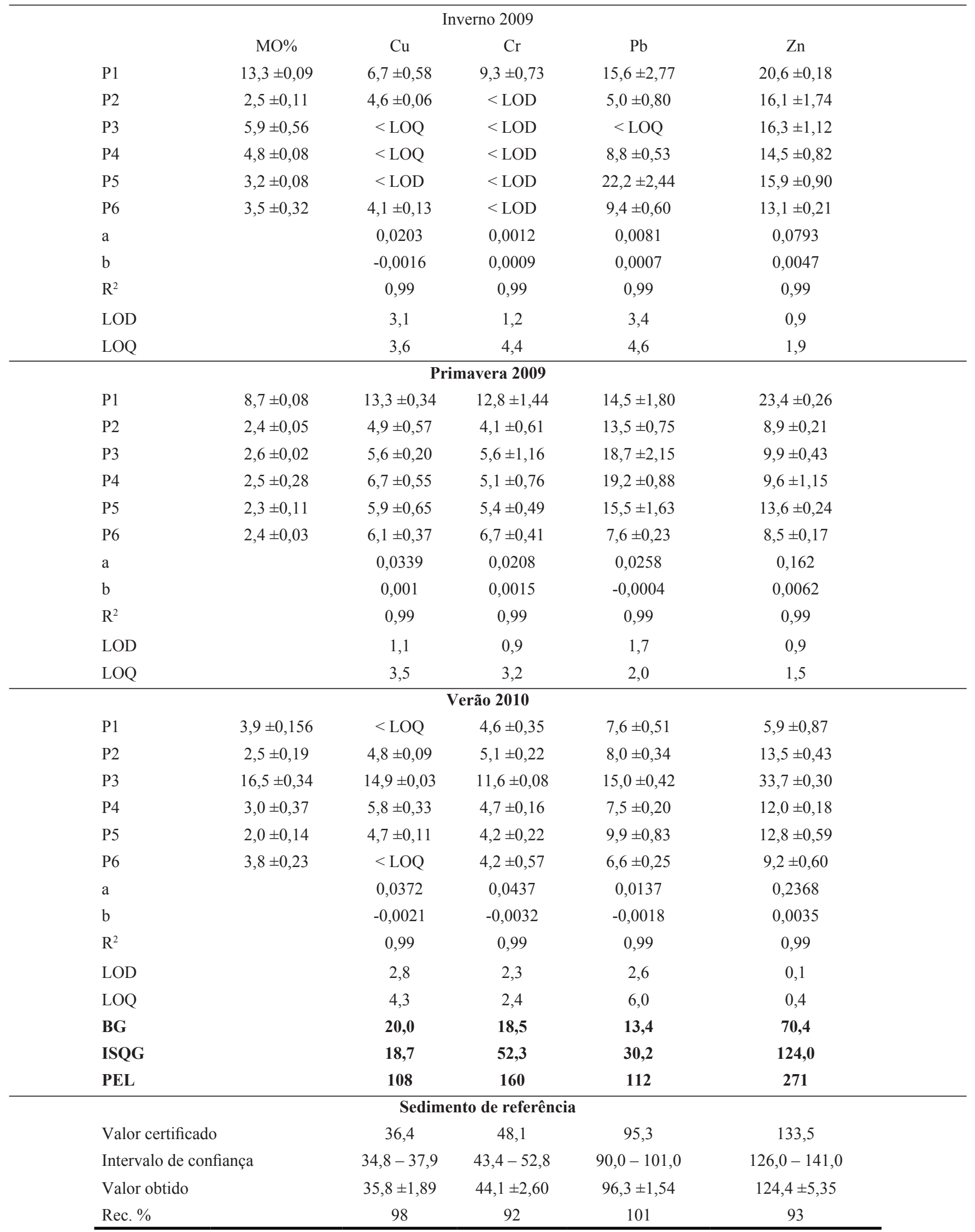


O controle de qualidade dos resultados obteve valores de recuperações entre 92 a $101 \%$ para todos os metais nas três campanhas amostrais. Segundo Jesus et al. (2004) fatores de recuperação entre 75 a $110 \%$ são considerados satisfatórios (Tabela 2).

A distribuição granulométrica dos sedimentos da região demonstra que o ponto 1 , no inverno e na primavera e, o ponto 3 no verão, apresentaram maior percentual de teor de finos (fração < $63 \mu \mathrm{m}$ ) (Tabela 3).

Lemes et al. (2003) aponta que o tamanho da partícula e grau de cristalinidade dos argilominerais influencia a sorção e a retenção de contaminantes na superfície das partículas. Comparando os níveis dos metais encontrados na fração do sedimento $<63 \mu \mathrm{m}$, observa-se que quanto maior o percentual dessa fração maior a concentração dos elementos estudados, exceto no caso do chumbo (Fig. 2).

Dos metais estudados, o $\mathrm{Zn}$ e o $\mathrm{Pb}$ destacam-se em relação aos demais na maioria dos pontos em todas as estações. Quando se trata do zinco, tal situação está em acordo com os resultados apresentados por Niencheski et al. (2002), que o aponta como elemento majoritário em seu estudo. As concentrações de $\mathrm{Pb}$ encontradas nos pontos estudados podem ser associadas à atividade pesqueira exercida na região durante o ano e também pelo fato dos pontos de coleta situarem-se em zonas de contribuições antrópicas.

$\mathrm{O} \mathrm{Cu}$ obteve seu maior índice no verão no ponto 3 , porém apresentou valores abaixo do limite de quantificação (LOQ) nos pontos 1 e 6 . No inverno o cromo foi detectado somente no ponto 1 , quando os teores de matéria orgânica foram elevados. Na primavera e no verão ocorreu a presença de $\mathrm{Cr}$ em todos os pontos. No ponto 1 foram encontrados valores de $\mathrm{Cu}$ e Cr próximos aos obtidos por Santos et al., (2003) em estudo realizado em setembro de 2001 para a mesma área situada no Canal São Gonçalo.

Observou-se nesta avaliação, que existe uma relação entre os níveis de metais e o aumento de matéria orgânica, bem como o teor de finos, o que explica a maior concentração de metais encontrada no ponto 3 no verão, e no ponto 1 nas demais estações com exceção do $\mathrm{Pb}$, que não foi possível estabelecer uma relação com os parâmetros estudados.

Pode-se concluir neste estudo que, embora os pontos de amostragem sejam localizados em zonas de contribuições antrópicas, o Saco do Laranjal não é considerado impactado pelos metais analisados segundo as referências de qualidade do sedimento descritas.

Tabela 3 - Distribuição granulométrica em \%.

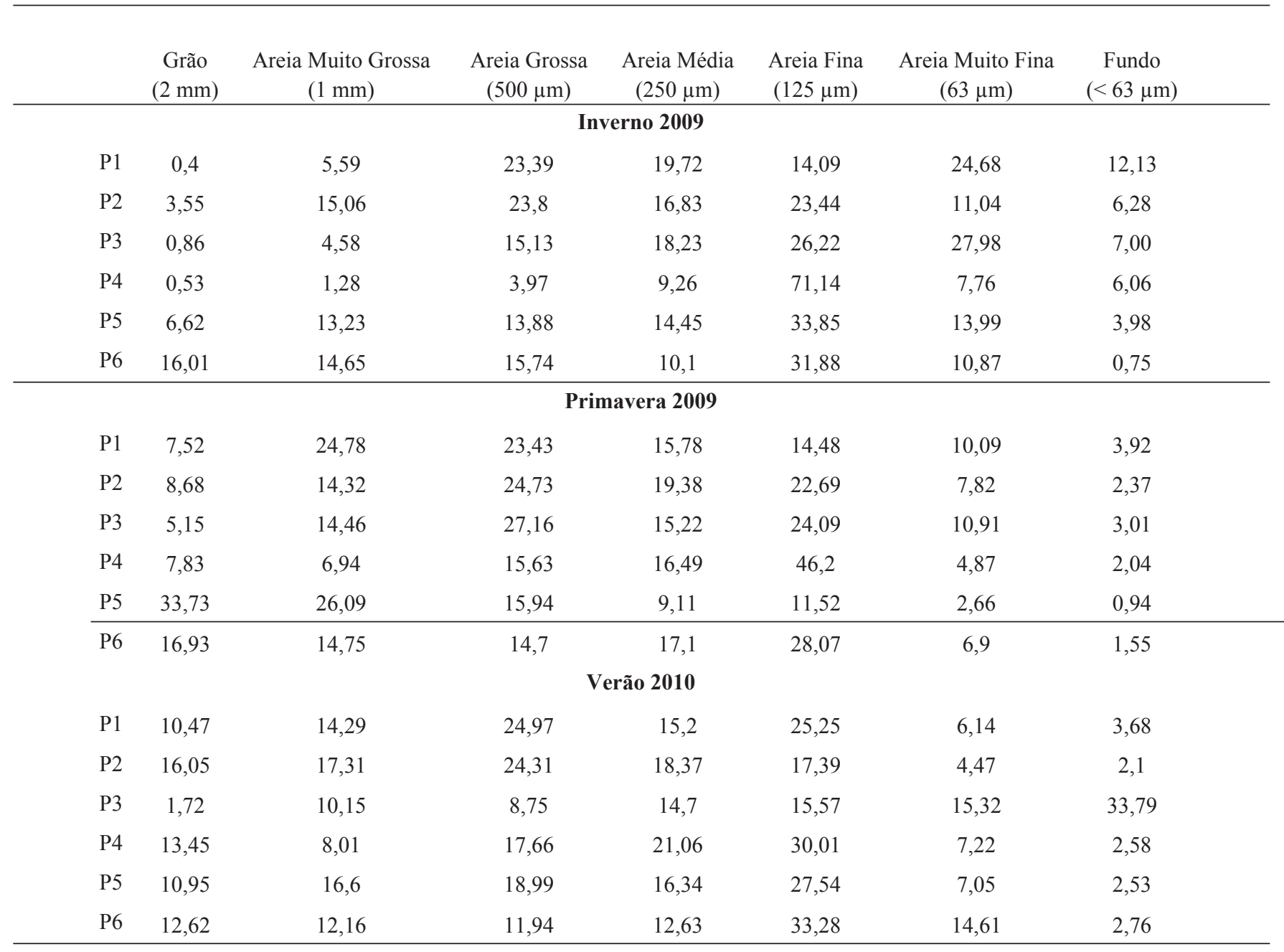




\section{Inverno}

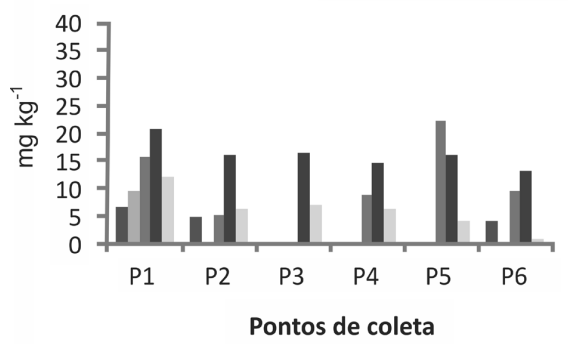

\section{Primavera}

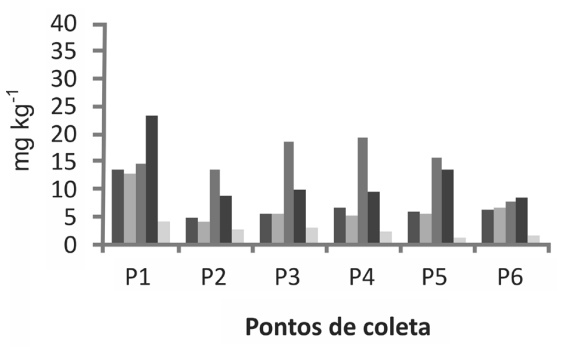

\section{Verão}

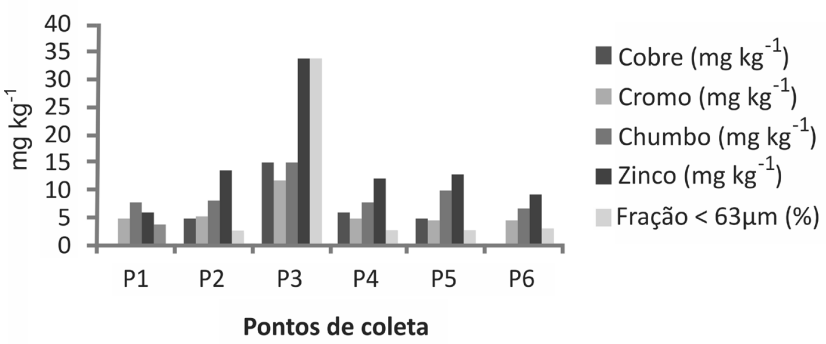

Figura 2. Comparação entre metais em $\mathrm{mg} \mathrm{Kg}^{-1}$ e teor de finos em \% (fração $<63 \mu \mathrm{m}$ ) nos períodos estudados.

\section{AGRADECIMENTOS}

Ao GPCA (Grupo de Pesquisas em Contaminantes Ambientais) e ao Instituto Federal Sul-rio-grandense por bolsa concedida.

\section{REFERÊNCIAS}

APHA, Standard Methods for the Examination of Water and Wastewater, $21^{\circ}$ edition, 2005.

CASTRO, S. V., 2006, Efeitos de metais pesados presentes na água sobre a estrutura das comunidades bentônicas do Alto Rio das Velhas-MG. Tese de Mestrado. Universidade Federal de Minas Gerais - UFMG. 110p.

CCME EPC- 98E, Canadian Sediment Quality Guidelines for the protection aquatic life, 1999.

ESTEVES, F. A., 1988, Fundamentos de Limnologia. Rio de Janeiro: Interciência: FINEP, 575p.

HORTELlANI, M. A., SARKIS, J. E. S., ABESSA, D. M. S., SOUSA, E. C. M., 2008, Avaliação da contaminação por elementos metálicos dos sedimentos do Estuário Santos - São Vicente. Quim. Nova., 31(1): 10-19.

IUPAC - International Union of Pure and Applied Chemistry, 1997, Chemistry Compendium of Chemical Terminology 2nd Edition.

JESUS, H. C., COSTA, E. A., MENDONÇA, A. S. F., ZANDONADE, E., 2004, Distribuição de metais pesados em sedimentos do sistema estuarino da Ilha de Vitória-ES. Quim. Nova., 27(1): 378-386

LACERDA, L. D.; MARINS, R. V., 2006, Geoquímica de sedimentos e o monitoramento de metais na plataforma continental nordeste oriental do Brasil. Geochim. Bras., Rio de Janeiro, 20(1): 123135.

LEMES, M. J. L., FIGUEIREDO FILHO, P. M., PIRES, M. A. F., 2003, Influência da mineralogia dos sedimentos das bacias hidrográficas dos rios Mogi-Guaçu e Pardo na composição química das águas de abastecimento público. Quim. Nova., 26(1): 13-20.

MIRLEAN, N. ANDRUS, V. E. BAISCH, P., 2003, Mercury pollution sources in sediments of Patos Lagoon Estuary, Southern Brazil. Mar. Pollut. Bull., 46(3): 331-334. DOI:10.1016/s0035326X(02)00404-6.

NIENCHESKI, L. F. H., BARAJ, B., FRANÇA, R. G., MIRLEAN, N., 2002, Lithium as a normaliser for assessment of anthropogenic metal contamination of sediments of the southern area of the Patos Lagoon. Aquat. Ecosyst. Health Manage., 5(4): 473-483. DOI: 10.1080/1463498029000197 7

RAMB - Relatório de qualidade ambiental da cidade de pelotas de 2003. Disponível em: <http://www.pelotas.com.br/sqa/ ramb2003.html> Acesso em: 18 nov. 2003.

SANTOS, I. R., BAISCH, P., LIMA, G. T. N. P., 2003, Metais pesados em sedimentos superficiais da Lagoa Mirim, fronteira Brasil-Uruguai. Geochim. Bras., 17(1): 37-47.

SUGUIO, K., 1973, Introdução à sedimentologia, São Paulo, 317p. TEÓDULO, M. J. S., LIMA, E. S., NEUMANN, V. H. M. L., LEITE, P. R. B., SANTOS, M. L. F. S., 2003, Comparação de métodos de extração parcial de metais traço em solos e sedimentos de um estuário tropical sob a influência de um complexo industrial portuário, Pernambuco Brasil. Estudos Geológicos, 13: 23-34.

WINDOM, H. L., NIENCHESKI, L. F., SMITH JR, R. G., 1999, Biogeochemistry of nutrients and trace metals in the estuarine region of the Patos Lagoon (Brazil). Estuar. Coast. Shelf Sci., 48: 113-123.

TORRES, R. F., LACERDA, L. D., AGUIAR, J. E., 2008, Biodisponibilidade de $\mathrm{Cu}$ e $\mathrm{Pb}$ em sedimentos de um canal de maré afluente do estuário do Jaguaribe - Ce. III Congresso Brasileiro de Oceanografia - CBO'2008, I Congresso IberoAmericano de Oceanografia - I CIAO, Fortaleza, Brasil. 